\title{
The Effect of Duration of Both Stages of Incremental Genetic Programming on its Efficiency of Evolution of Snakebot
}

\author{
Naoki Mukosaka, Yasuto Nishiwaki, Ivan Tanev, Katsunori Shimohara \\ Graduate School of Science and Engineering, Doshisha University, \\ 1-3 Tatara-Miyakotani, Kyotanabe, Kyoto 610-0321, Japan \\ E-mail: mukosaka2012@sil.doshisha.ac.jp,nishiwaki2012@sil.doshishsa.ac.jp,itanev@mail.doshisha.ac.jp, \\ kshimoha@mail.doshisha.ac.jp \\ www.doshisha.ac.jp
}

\begin{abstract}
The objective of our work is to investigate the optimal combination of durations of both stages of incremental Genetic Programming (IncGP) on the simulated Snake-like robots (Snakebots). The obtained results suggest that the number of generations cannot be used as criteria for separating the two stages of IncGP. We hypothesized that qualitative criterion, such as the emergence of smooth sidewinding locomotion at the first stage of evolution could be used instead, and conducted additional experiments that proved its worthiness.
\end{abstract}

Keywords: Genetic Programming, Snake-like Robot, Incremental Genetic Programming, Evolution.

\section{Introduction}

Compared to the wheeled and legged vehicles, the wheel-less, limbless Snake-like robots (Snakebots) are more robust and adaptable to both the environmental changes and partial damage. Some of the useful features of Snakebots include smaller size of the cross-sectional area, stability, ability to operate in rugged terrain, good traction, high redundancy, and complete sealing of the internal mechanisms ${ }^{1}$. Due to the morphological complexity of Snakebots, an automated approach of developing their controller via Genetic Programming (GP) is often used ${ }^{1}$.

However, the evolution of Snakebot from scratch via GP is rather inefficient when the bot is situated in a challenging environment. The main reason for such inefficiency is that the evolution needs to simultaneously discover both (i) the generic locomotion traits (that are common for all well-moving bots, regardless of the environment) and (ii) the specific traits that are needed to overcome the obstacles in these challenging terrains.

In order to improve the efficiency of such an evolution, in our previous work ${ }^{2}$, we divided the task of evolving the locomotion of simulated Snakebot in challenging terrain into two consecutive, simpler subtasks, implemented as two consecutive stages of an incremental Genetic Programming (IncGP): at the first stage we applied GP to evolve a pool of bots that are able to move fast in a smooth, open terrain. Then, during the second stage, we used this pool to seed the 
initial population of the bots that are further subjected to evolution in the challenging environment. We demonstrated that the two-staged incremental evolution via IncGP, consisting of two stages of 40 generations each, features a much improved efficiency over the canonical $\mathrm{GP}^{2}$.

The objective of our current work is to investigate the optimal combination of durations of both stages of incGP. We assume a fixed runtime budget of evolution, corresponding to 80 generations overall. Therefore, our experiments involved evolution of Snakebot via incGP with 7 combinations of durations of both stages of evolution: from 10 generations of the first stage and 70 generations of the second one, to 70 generations of the first and 10 generations of the second one (i.e., 10 and 70,20 and 60, 30 and 50,.., and, finally, 70 and 10, respectively).

\section{Simulated Snakebot}

In our work we used the Open Dynamics Engine ${ }^{3}$ (ODE) physics library to simulate the articulated rigid body dynamics of the Snakebot ${ }^{2}$. ODE allows accounting for all forces, resulting from friction, gravity, actuators, and collisions between the segments of the simulated bot, and the environment. The Snakebot is identical to the one as used in our prior work $^{1,2,4 \text {, and } 5}$. It is simulated as 15 identical spherical morphological segments ("vertebrae"). Pairs of nearby segment are linked by universal (Cardano) joints and all joints are powered by two (horizontal and vertical) identical actuators ("muscles").

In this study we use IncGP to evolve such motion patterns of the segments of the bot that result in fastest speed of locomotion of the bot. Considering the representation of Snakebot, this task could be rephrased as developing the temporal patterns of desired turning angles of horizontal and vertical actuators of each segment, that result in fastest overall locomotion of Snakebot. The proposed representation of Snakebot as a homogeneous system comprising identical morphological segments is intended to significantly reduce the size of the search space of the IncGP (as we need to evolve the functionality of one pairs of actuators only) and to facilitate favorable scalability characteristics of the latter.

\section{Simulation Setup}

We employed IncGP in two consecutive evolutionary stages as follows:
- First, we implemented 30 independent runs of canonical GP to evolve the Snakebot in a smooth terrain for $\mathrm{N}$ generations $(\mathrm{N}=10,20,30, \ldots, 70)$, and created a pool of 6 best-of-run, fast moving Snakebots for each corresponding value of N.

- In the second stage of IncGP, we conducted additional 30 independent runs, (for $80-\mathrm{N}$ generations) to evolve the bot, situated in a challenging terrain. The initial population of each of these runs is seeded with the 6 fast moving bots, obtained in the first stage of GP, plus additional 194 randomly created bots. For the second stage of IncGP, we considered two cases of challenging environment, and one case of partial damage, as elaborated below.

The considered two cases of challenging terrain include (i) a "pen" of walls (which surrounds the bot from all four sides), and (ii) multiple small immobile (i.e., fixed to the ground) obstacles. In the first case, the height of the wall is automatically increased each time the bot overcomes it. Therefore, the maximum height of the cleared wall (at the end of evolutionary run) could be viewed as a measure of the ability of the bot to overcome walls. In the second case, the average speed of the bot (calculated from the travelled center of the gravity of the latter and the time of the trial) is considered as a measure of the ability of bot to overcome obstacles.

In addition, we conducted experiments with IncGP on evolutionary adaptation of the bot to its partial damage to 6 (out of 15) segments. In this case, we assumed that the damage renders the joint at the tailside of the affected segment completely immobile.

The main parameters of IncGP are summarized in Table 1. We used our in-house DOM/XML-based implementation of $\mathrm{GP}^{6}$.

Table. 1. Main parameters of IncGP

\begin{tabular}{ll}
\hline Category & Value \\
\hline Genotype & $\begin{array}{l}\text { Patterns of the turning angles of: } \\
\text { (i) horizontal actuator, and } \\
\text { (ii)Vertical actuator }\end{array}$ \\
Population size & 200 individuals \\
Selection & Binary Tournament, ratio: 0.1 \\
Elitism & Reproduction ratio: 0.9 \\
Mutation & Best 4 individuals \\
\hline
\end{tabular}




\section{Experimental Results}

The experimental results of each of cases are shown in Fig.1, Fig.2, and Fig.3, respectively.

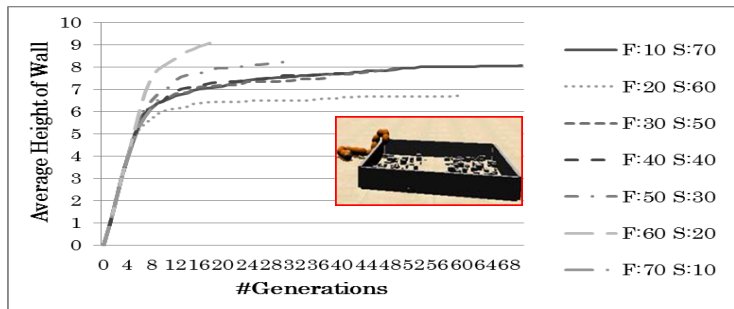

Fig. 1. Dynamics of the height of walls overcame by Snakebot in the second stage of IncGP. Unit on the ordinate corresponds to $1 / 4^{\text {th }}$ of the diameter of the spherical segments of the bot.

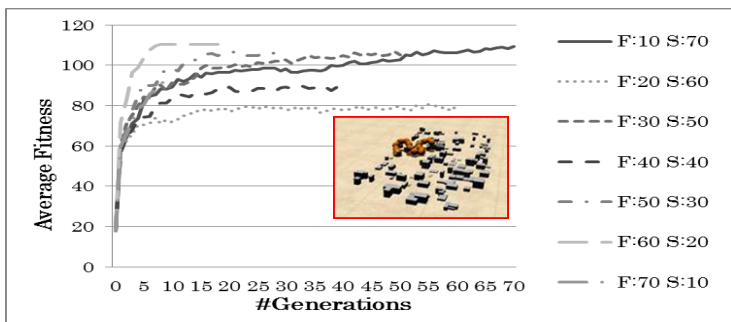

Fig. 2. Fitness convergence of evolving Snakebot in challenging environment with many small obstacles

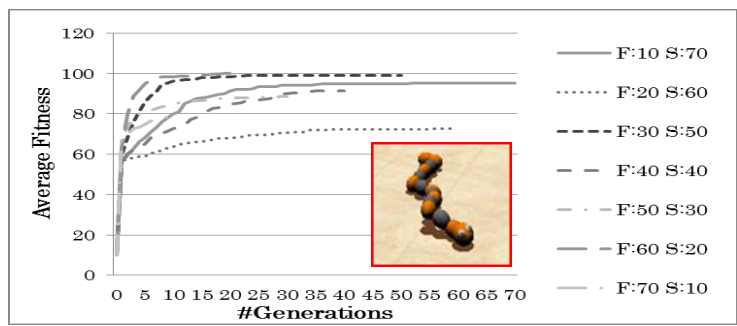

Fig. 3. Fitness convergence of evolving Snakebot with a failure of 6 (out of 15) segments

The statistical results including the complexity of genotype (in number of tree nodes) and fitness value are summarized in Table 2.

\section{Discussion}

The experimental results suggest that neither the number of generations nor the obtained fitness value at the first stage of IncGP can be used as criteria for optimization of the duration of the two stages of IncGP. Moreover, as the results in Table 2 indicate, there is no correlation between (i) the complexity of the genotype of the Snakebots (in tree nodes) evolved in the first stage, and (ii) the performance of the best-evolved bot at the second stage of IncGP.

Table. 2. The complexity of genotypes and fitness values in IncGP. $F N_{a v r}$ and $F F_{a v r}$ denote the average number of tree nodes and the average fitness in the first stage of IncGP, respectively. $H o f W_{a v r}$ is the height of wall that the bot overcame in the second stage of IncGP. FMO avr and D6F avr are the average fitness values in the second stage for the cases of environment with multiple small obstacles and damage to six segments, respectively.

\begin{tabular}{cllllll}
\hline $\begin{array}{l}\text { Duration of First } \\
\begin{array}{l}\text { (F) and second (S) } \\
\text { stages of IncGP }\end{array}\end{array}$ & $\boldsymbol{F N}_{\boldsymbol{a v r}}$ & $\boldsymbol{F} \boldsymbol{F}_{\boldsymbol{a v r}}$ & $\boldsymbol{H o f} \boldsymbol{W}_{\boldsymbol{a v r}}$ & $\boldsymbol{F M O _ { a v r }}$ & $\boldsymbol{D 6 F}_{\boldsymbol{a v r}}$ \\
\hline F:10 S:70 & 54.67 & 87.3 & 8.067 & 109 & 95.17 \\
F:20 S:60 & 74 & 89.5 & 6.7 & 79.8 & 72.6 \\
F:30 S:50 & 82.67 & 103 & 7.933 & 105 & 99.2 \\
F:40 S:40 & 95.83 & 103 & 7.7 & 89.3 & 91.53 \\
F:50 S:30 & 93.17 & 107 & 8.233 & 106 & 88.63 \\
F:60 S:20 & 68 & 112 & 9.133 & 110 & 100.1 \\
F:70 S:10 & 77.67 & 100 & 6.667 & 91.4 & 79.07 \\
\hline
\end{tabular}

Therefore, we hypothesize that the criteria for terminating the first stage of IncGP and initializing the second one might not be necessarily a quantitative one. Rather, an aesthetic, intuitive, subjective, and informal evaluation from the human about the presence of generic locomotion would suffice for the switch between the both stages of IncGP.

In order to verify this hypothesis, conducted additional experiments intended to estimate the importance of human-judged evaluation of the Snakebots as follows:

- First, we implemented 6 independent runs of canonical GP to evolve the Snakebot in a smooth terrain for $\mathrm{N}$ generations $(\mathrm{N}=10,20,30, \ldots, 70)$, and created a pool of 6 fast moving Snakebots.

- In the second stage of IncGP, we conducted additional 6 independent runs (for $80-\mathrm{N}$ generation) of evolution of the bot, situated in the challenging terrain. For the second stage of IncGP, we only considered the case of "pen" of walls.

We conducted the above mentioned experiments four times for each combination of durations of both stages. We looked at the visual phenotypic traits of the best-of-run, fast moving generic bots obtained at the first stage of IncGP. We noticed that the number of bots 
that move smoothly via sidewinding, and feature sinusoidal shape, correlates well with the degree of success at the second stage of IncGP. These results are summarized in Fig.4. As figure illustrates, the quality of the bots (i.e., the maximum height of the wall it overcomes), evolved in the second stage of IncGP, increases with the increase of the number of smooth sidewinders from one to four in the pool of the six bots used for seeding of the second stage of IncGP. Additional increase of the number of smooth sidewinders to five and six is associated with a decrease of the quality in bots evolved at the second stage of IncGP. The reason for such an anomaly is that achieving five or six smooth sidewinding bots at the first stage usually requires an evolution for too many generations $\mathrm{N}$, and the remaining budget of the number of generations $(80-\mathrm{N})$ for the second stage does not allow enough time for the bot to learn how to overcome tall walls. From another perspective, the nonmonotonous nature of the dependency illustrated in Fig.4 suggests that there is an optimal point in configuring the IncGP, and that our consideration of the visual, qualitative phenotypic traits of the evolved Snakebots facilitated the discovery of this point.

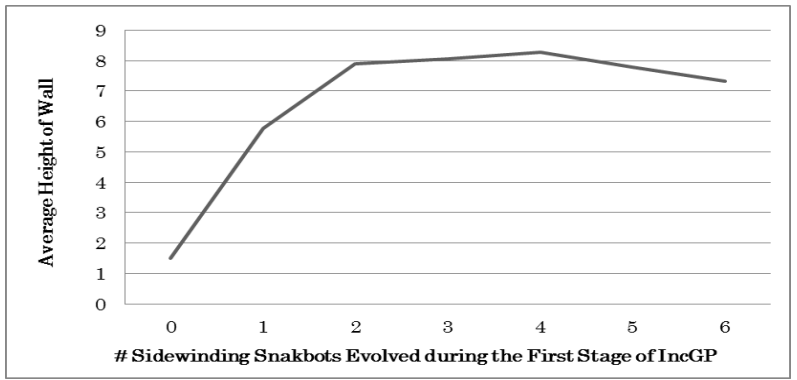

Fig. 4. Maximum height of walls that are overcame by Snakebot in second stage as a function of the number of smooth sidewinding bots evolved in first stage of IncGP.

\section{Conclusion}

In this paper, we investigated the effect of duration of both stages of incremental genetic programming on its efficiency of evolution of Snakebot. We discovered that the success of the second stage does not depend on the quantitative metrics (e.g., number of generations, fitness value, complexity of genotype, etc.) pertinent to the first stage of evolution of the bot. Consequently, we could conclude that these quantitative metrics could not be used as reliable criteria to decide the switch from the first stage of IncGP to the second one.
We experimentally proved our hypothesis that the smooth sidewinding with an aesthetically wellperceived sinusoidal shape of the bot can be considered as phenotypic traits of the generic locomotion gaits that are very important for the success of the second stage of evolution. Once the evolution discovers these generic gaits, it could terminate the first stage of evolution and switch its efforts to the second stage. During the latter, these generic gaits could be enhanced further by incorporating additional features that are specifically needed to deal with the surrounding challenging terrain. This discovery opens up the opportunity for an original way of incorporating interactive evolution into IncGP in a way that allows a human to subjectively influence the optimal breakdown between the both stages of IncGP. We are planning to investigate this opportunity in our future work.

\section{Acknowledgement}

The first author would like to thank the members of the Laboratory of Socioinformatics at Doshisha University for their support of this study.

\section{References}

1. I. Tanev, T. Ray and A. Buller, Automated Evolutionary Design, Robustness and Adaptation of Sidewinding Locomotion of Simulated Snake-like Robot, IEEE Transactions on Robotics, Vol.21, Number 4, August 2005, pp.632-645

2. N. Mukosaka, I. Tanev, and K. Shimhara, Performance of Incremental Genetic Programming on Adaptability of Snake-like Robot, 17th Asia Pacific Symposium on Intelligent and Evolutionary Systems, IES2013, Volume 24, 2013, pp.152-157

3. R. Smith, Open Dynamics Engine, URL: http://ode.org/

4. T. Kuyucu, I. Tanev and K. Shimohara, Incremental Genetic Programming Via Genetic Transpositions For Efficient Coevolution Of Locomotion And Sensing Of Simulated Snake-Like Robot Proceedings of the 20th he European Conference on Artificial Life (ECAL-2011), 8th - 12th August 2011, Paris, France, pp. 439-446

5. I. Tanev, T. Kuyucu, and K. Shimohara, On the Cumulative Effect of Bloat and Genetic Transposition on the Efficiency of Incremental Evolution of Snake-like Robot Genetic and Evolutionary Computation Conference, GECCO-2012, July 7-11, 2012, Philadelphia, USA, pp.161-168

6. I. Tanev. Dom/xml-based Portable Genetic Representation of the Morphology, Behavior and Communication Abilities of Evolvable Agents, Artificial Life and Robotics, 2004, 8:52-56 